PROCEEDINGS OF THE

AMERICAN MATHEMATICAL SOCIETY

Volume 131, Number 6, Pages 1935-1942

S 0002-9939(02)06728-X

Article electronically published on October 1, 2002

\title{
CHARACTERISTIC CLASSES AND TRANSFER RELATIONS IN COBORDISM
}

\author{
M. BAKURADZE, M. JIBLADZE, AND V. V. VERSHININ
}

(Communicated by Paul Goerss)

\begin{abstract}
Decompositions of products of the Ray elements by free generators of small dimensions in the symplectic cobordism ring are obtained. In particular it is stated that most of the $4 n$-dimensional generators, for $n$ small, after multiplication by the Ray elements $\phi_{i}, i \geqslant 0$, land in the ideal generated by Ray elements of low dimension.
\end{abstract}

\section{INTRODUCTION}

Immediately after its first appearence in the papers of J. Milnor [12] and S. P. Novikov [14, the symplectic cobordism attracted attention of many homotopy theorists. However, unlike the cobordism theories corresponding to other classical Lie groups - e.g. nonoriented $(\mathrm{O}(n))$, oriented $(\mathrm{SO}(n))$ and complex $(\mathrm{U}(n))$ - the structure of its coefficient ring remains largely unknown. In the study of symplectic cobordism various methods have been applied: the classical Adams spectral sequence [14, the Adams-Novikov spectral sequence [18, 20], the Atiyah-Hirzebruch spectral sequence [16], the use of characteristic classes and generalizations of formal groups [15, 5], and cobordism with singularities [19]. In this paper we apply the transfer maps to the study of the symplectic cobordism ring. Transfers first appeared in group theory at the beginning of the twentieth century in the works of I. Schur, as natural maps from the abelianization of a group to abelianizations of its subgroups, and then were generalized to other homologies and cohomologies of groups (see, e.g. 4]). In the work of J. C. Becker and P. H. Gottlieb [2], transfer maps were constructed as morphisms in the stable category and since then have been widely used in homotopy theory.

Since the work of S. P. Novikov [14] it is known that rationally the symplectic cobordism ring $M \mathrm{Sp}_{*}$ is isomorphic to the polynomial ring on an infinite number of generators which appear in dimensions $4 n$ for all natural $n$. In the torsion part the key role is played by the family of elements $\phi_{i} \in M \mathrm{Sp}_{8 i-3}$ of order 2 defined by Nigel Ray [15].

Received by the editors June 10, 2001 and, in revised form, January 15, 2002.

2000 Mathematics Subject Classification. Primary 55N22, 55R12.

The first author was supported by the CRDF grant \#GM1-2083 and by the Abdus Salam International Centre for Theoretical Physics, Trieste, Italy.

The second author was supported by the TMR research network ERB FMRX CT-97-0107 and the INTAS grant \#93-3218-EXT.

The third author was supported in part by the French-Russian Program of Research EGIDE (dossier No 04495UL). 
Most of the relations between the Ray elements $\phi_{i}$ and free generators in the torsion part of the symplectic cobordism ring up to dimension 32 [18, 19] can be conventionally subdivided into three types: to the first type correspond relations which mainly follow from relations in the integral part coming from $M \mathrm{Sp}_{4 n}$. In more detail, relations of the first type have form $(x+y) \phi_{i}=0$, and these relations follow from the fact that the sum of free generators $x+y$ is divisible by 2 , whereas the Ray elements are of order 2. Relations of the second type have form $z \phi_{i}=0$ where $z$ is again a $4 n$-dimensional generator from the free part. The aim of the present paper is to elucidate origins of relations of the third type mentioned in the abstract above.

Let $\zeta$ denote the universal $\operatorname{Sp}(1)$-bundle. Then $\zeta_{1} \otimes_{\mathbb{C}} \zeta_{2} \otimes_{\mathbb{C}} \zeta_{3}$ is a symplectic bundle over $B \operatorname{Sp}(1) \times B \operatorname{Sp}(1) \times B \operatorname{Sp}(1)$. Also $\zeta_{1} \otimes_{\mathbb{C}} \zeta_{2}^{2}$ and $\zeta_{1} \otimes_{\mathbb{R}} \zeta_{2}$ are symplectic bundles over $B \operatorname{Sp}(1) \times B \operatorname{Sp}(1)$.

Section 2 is devoted to the calculation of transfers [1, 6, 2, 11]. In Section 3 we prove the following main result:

Theorem 1.1. Let $x_{i}=p_{1}\left(\zeta_{i}\right), i=1,2$, be the first Conner-Floyd symplectic Pontyagin class. Let $\phi_{j}, j \geqslant 0$, be the Ray elements, and let $n$ be such that $M \mathrm{Sp}_{4 m}$ is torsion free for $m \leqslant 2 n-1$. Then:

a) the element $\phi_{j} p_{1}\left(\zeta_{1} \otimes_{\mathbb{C}} \zeta_{2}^{2}\right)$ is divisible by $\phi_{0} x_{1}+\phi_{1} x_{1}^{2}+\ldots+\phi_{[n / 2]} x_{1}^{2[n / 2]}$;

b) $\phi_{j} p_{1}\left(\zeta_{1} \otimes_{\mathbb{R}} \zeta_{2}\right)=0$

in the ring $M \mathrm{Sp}^{*}\left(\mathbb{H} P(n)^{2}\right)=M \mathrm{Sp}^{*} \llbracket x_{1}, x_{2} \rrbracket /\left(x_{i}^{n+1}\right)$.

In Section 4 we will see that in terms of the coefficients $a_{k l m}$ of the first ConnerFloyd symplectic Pontryagin class

$$
p_{1}\left(\zeta_{1} \underset{\mathbb{C}}{\otimes} \zeta_{2} \underset{\mathbb{C}}{\otimes} \zeta_{3}\right)=\sum_{k+l+m \geqslant 1} a_{k l m} p_{1}^{k}\left(\zeta_{1}\right) p_{1}^{l}\left(\zeta_{2}\right) p_{1}^{m}\left(\zeta_{3}\right),
$$

the structure of $M \mathrm{Sp}_{4 k}, k \leqslant 4$, can be interpreted as follows:

\begin{tabular}{|l|c|c|}
\hline$k$ & $M \mathrm{Sp}_{4 k}$ & generators \\
\hline 1 & $\mathbb{Z}$ & $a_{011}$ \\
\hline 2 & $\mathbb{Z}+\mathbb{Z}$ & $a_{012}, a_{111}$ \\
\hline 3 & $\mathbb{Z}+\mathbb{Z}+\mathbb{Z}$ & $a_{022}, a_{011} a_{111}, a_{211}$ \\
\hline 4 & $\mathbb{Z}+\mathbb{Z}+\mathbb{Z}+\mathbb{Z}+\mathbb{Z}$ & $a_{014}, a_{011} a_{211}, a_{122}, a_{111}^{2}, 2 y_{4}$ \\
\hline
\end{tabular}

Then Theorem 1.1 implies

Corollary 1.2. For $i \geqslant 0$ one has:

a) $\phi_{i} a_{001}=\phi_{i} a_{012}=\phi_{i} a_{022}=\phi_{i} a_{014}=0$;

b) $\phi_{i} a_{111}$ and $\phi_{i} a_{122}$ belong to the ideal $\phi_{0} M \mathrm{Sp}^{*}$;

c) $\phi_{i} a_{211}$ belongs to the ideal $\phi_{0} M \mathrm{Sp}^{*}+\phi_{1} M \mathrm{Sp}^{*}$.

Relations of Corollary 1.2 imply that multiplication by the elements $\phi_{i}, i \geqslant 0$, carries most of the low-dimensional generators from the free part of $M \mathrm{Sp}_{4 n}$ to the ideal generated by the elements $\phi_{0}$ and $\phi_{1}$. 


\section{Preliminaries and CAlCUlations With transfer}

Let $\xi$ and $\Lambda$ be, respectively, the universal U(1)-bundle and the universal Spin(3)bundle. Thus the sphere bundle of $\Lambda$ is $\pi: B \mathrm{U}(1) \rightarrow B \mathrm{Sp}(1)$, and one has

$$
\begin{aligned}
\pi^{*}(\zeta) & =\xi+\bar{\xi}, \\
\pi^{*}(\Lambda) & =\xi^{2}+\mathbb{R}, \\
\zeta \underset{\mathbb{H}}{\otimes} \zeta & =\Lambda+\mathbb{R},
\end{aligned}
$$

where $\zeta$ is the universal $\mathrm{Sp}(1)$-bundle as above. Let $\mathrm{N}$ denote the normalizer of the torus $\mathrm{U}(1)$ in $\mathrm{Sp}(1)$. The classifying space $B \mathrm{~N}$ coincides with the orbit space of the complex projective space $\mathbb{C} P(\infty)$ under the free involution $I$, which acts via

$$
I:\left[z_{0}, z_{1}, \ldots\right] \mapsto\left[-\bar{z}_{1}, \bar{z}_{0}, \ldots\right]
$$

in homogeneous coordinates.

The bundle $p: B \mathrm{~N} \rightarrow B \mathrm{Sp}(1)$ coincides with the projective bundle of $\Lambda$ and one has the canonical splitting

$$
p^{*}(\Lambda)=\mu+\nu
$$

defined by projectivisation $p$, where $\mu$ and $\nu$ denote real plane and line bundles, respectively. Of course for the double covering $q: B \mathrm{U}(1) \rightarrow B \mathrm{~N}$ one has $q^{*}(\mu)=\xi^{2}$ and $q^{*}(\nu)=\mathbb{R}$.

Let $\tau_{\pi}$ and $\tau_{p}$ be the transfer maps of the bundles $\pi$ and $p$ [2, 6, 11]. The next lemma follows from [7.

Lemma 2.1. $\pi^{*} \tau_{\pi}^{*}=1+I^{*}$ and $\pi^{*} \tau_{p}^{*}=q^{*}$.

The next lemma follows from the definitions.

Lemma 2.2. $\left(\xi_{1} \xi_{2}^{2}+\bar{\xi}_{1} \bar{\xi}_{2}^{2}\right) !=\left(\xi_{1}+\bar{\xi}_{1}\right) \otimes_{\mathbb{R}} \mu$, where '(-)!' denotes the Atiyah transfer for the double covering $1_{B \mathrm{U}(1)} \times q$.

Consider the map $f: B \mathrm{~N} \rightarrow B \mathbb{Z} / 2$ induced by the projection of $\mathrm{N}$ onto the Weyl group $\mathbb{Z} / 2$ and let $\tau_{1 \times q}^{*}$ be the transfer homomorphism for the above double covering $1_{B \mathrm{U}(1)} \times q$.

Lemma 2.3. For some elements $\alpha_{i} \in \widetilde{M S p}^{*}(B \mathbb{Z} / 2)$ the following formula holds:

$$
\tau_{1 \times q}^{*}\left(p_{1}\left(\xi_{1} \xi_{2}^{2}+\bar{\xi}_{1} \bar{\xi}_{2}^{2}\right)\right)=p_{1}\left(\left(\xi_{1}+\bar{\xi}_{1}\right) \underset{\mathbb{R}}{\otimes} \mu\right)+\sum_{i \geqslant 0} f^{*}\left(\alpha_{i}\right) p_{2}^{i}\left(\left(\xi_{1}+\bar{\xi}_{1}\right) \underset{\mathbb{R}}{\otimes} \mu\right) .
$$

Proof. Taking into account Lemma 2.2 the proof follows from the following formula [17]: let $q$ be the double covering $q: X \rightarrow B$, let $\eta \rightarrow X$ be the symplectic line bundle, $\eta_{!} \rightarrow B$ the Atiyah transfer bundle, $\tau_{q}$ the transfer map of the covering $q$ and $f: X \rightarrow B \mathbb{Z} / 2$ the classifying map of the real line bundle associated with $q$. Then for some elements $\alpha_{i}$ from $\widetilde{M S p}^{*}(B \mathbb{Z} / 2)$ the following formula holds:

$$
\tau_{q}^{*}\left(p_{1}(\eta)\right)=p_{1}\left(\eta_{!}\right)+\sum_{i \geqslant 0} f^{*}\left(\alpha_{i}\right) p_{2}^{i}\left(\eta_{!}\right)
$$

Lemma 2.4. Let $\tau$ be the transfer of the sphere bundle of a $\operatorname{Spin}(3)$-bundle. Then $\phi_{j} \operatorname{Im} \tau^{*}=0, j \geqslant 0$. 
Proof. Of course it suffices to prove this for the universal $\operatorname{Spin}(3)$-bundle $\Lambda$, that is, $\phi_{j} \tau_{\pi}^{*}(a)=0$ for all $a \in M \mathrm{Sp}^{*}(B \mathrm{U}(1))$.

Let $\delta_{\pi}$ be the Boardman map [3]. Then as it is known from [2],

$$
\tau_{\pi}^{*}(a)=\delta_{\pi}\left(a e\left(\xi_{2}^{2}\right)\right) .
$$

Here $e\left(\xi_{2}^{2}\right)$ is the Euler class of the bundle $\xi_{2}^{2}$ which is the bundle of tangents along the fibers. Then from [13, 8, $\phi_{j} e\left(\xi_{2}^{2}\right)=0$. This proves Lemma 2.4

Recall from [13, 8, 9] that the bundle $\Lambda$ is $M$ Sp-orientable and the corresponding Euler class has the form

$$
e(\Lambda)=\phi_{0} p_{1}(\zeta)+\sum_{j \geqslant 1} \phi_{j} p_{1}^{2 j}(\zeta)
$$

The restrictions of $\pi$ and $p$ to the symplectic projective space $\mathbb{H} P(n)$ will be denoted by the same symbols. Total spaces of these bundles coincide, respectively, with the complex projective space $\mathbb{C} P(2 n+1)$ and with the orbit space $\mathbb{C} P(2 n+1) / I$ under the free involution $I$ which acts via

$$
\left[z_{0}, z_{1}, \ldots, z_{2 n}, z_{2 n+1}\right] \mapsto\left[-\bar{z}_{1}, \bar{z}_{0}, \ldots,-\bar{z}_{2 n+1}, \bar{z}_{2 n}\right]
$$

in homogeneous coordinates.

Proposition 2.5. $\phi_{j} \tau_{\pi \times 1}^{*}\left(p_{i}\left(r \xi \otimes_{\mathbb{R}} \zeta\right)\right)=0$ for $\pi \times 1=\pi \times 1_{B S p(1)}: B \mathrm{U}(1) \times$ $B \mathrm{Sp}(1) \rightarrow B \mathrm{Sp}(1)^{2}, j \geqslant 0$, and $i=1,2$.

Proof. In $M \mathrm{Sp}^{*}(B \mathrm{U}(1) \times B \mathrm{Sp}(1))=M \mathrm{Sp}^{*}(B \mathrm{U}(1)) \llbracket p_{1}(\zeta) \rrbracket$ one has $p_{i}\left(r \xi \otimes_{\mathbb{R}} \zeta\right)=$ $\sum_{k \geqslant 0} \omega_{k}^{(i)} p_{1}^{k}(\zeta)$. Then it follows from Lemma 2.4 that

$$
\phi_{j} \tau^{*}\left(\sum_{k \geqslant 0} \omega_{k}^{(i)} p_{1}^{k}(\zeta)\right)=\sum_{k \geqslant 0} \phi_{j} \tau^{*}\left(\omega_{k}^{(i)} p_{1}^{k}(\zeta)\right)=0 .
$$

\section{Proof of Theorem 1.1}

The bundle $\pi \times 1: \mathbb{C} P(2 n+1) \times \mathbb{H} P(n) \rightarrow \mathbb{H} P(n) \times \mathbb{H} P(n)$ coincides with the sphere bundle of the pullback of $\Lambda \rightarrow \mathbb{H} P(n)$ along the projection on the first factor $\mathbb{H} P(n) \times \mathbb{H} P(n) \rightarrow \mathbb{H} P(n)$. So taking into account the formula (2.5) we have to prove that

$$
\left(\pi \times 1_{\mathbb{H} P(n)}\right)^{*}\left(\phi_{j} p_{1}\left(\zeta_{1} \underset{\mathbb{C}}{\otimes} \zeta_{2}^{2}\right)\right)=0
$$

in $M \mathrm{Sp}^{*}(\mathbb{C} P(2 n+1) \times \mathbb{H} P(n))$. The transfer $\tau^{*}=\tau_{1 \times \pi}^{*}$ of the bundle $1_{\mathbb{C} P(2 n+1)} \times \pi$ is a composite of two transfers, namely

$$
\tau^{*}=\tau_{1}^{*}\left(\tau_{2}^{*}\right)
$$

where $\tau_{1}$ is the transfer of the bundle $1_{\mathbb{C} P(2 n+1)} \times q$ and $\tau_{2}$ is the transfer of $1_{\mathbb{C} P(2 n+1)} \times p$, where the bundles $p, \pi$ and $q$ are the bundles defined above; that is,

$$
\begin{aligned}
& 1 \times q: \mathbb{C} P(2 n+1) \times \mathbb{C} P(2 n+1) \rightarrow \mathbb{C} P(2 n+1) \times \mathbb{C} P(2 n+1) / I, \\
& 1 \times p: \mathbb{C} P(2 n+1) \times \mathbb{C} P(2 n+1) / I \rightarrow \mathbb{C} P(2 n+1) \times \mathbb{H} P(n) .
\end{aligned}
$$

Using (2.2) one obtains $\left(\xi_{1}+\bar{\xi}_{1}\right) \otimes_{\mathbb{C}} \zeta_{2}^{2}=\left(\xi_{1}+\bar{\xi}_{1}\right) \otimes_{\mathbb{R}}(\Lambda+\mathbb{R})$, hence

$$
p_{1}\left(\left(\xi_{1}+\bar{\xi}_{1}\right) \underset{\mathbb{C}}{\otimes} \zeta_{2}^{2}\right)=p_{1}\left(\left(\xi_{1}+\bar{\xi}_{1}\right) \underset{\mathbb{R}}{\otimes} \Lambda\right)+p_{1}\left(\xi_{1}+\bar{\xi}_{1}\right) .
$$


Applying Lemma 2.2 and Lemma 2.3 one obtains

$$
\tau_{1}^{*}\left(p_{1}\left(\xi_{1} \xi_{2}^{2}+\bar{\xi}_{1} \bar{\xi}_{2}^{2}\right)\right)=p_{1}\left(\left(\xi_{1}+\bar{\xi}_{1}\right) \underset{\mathbb{R}}{\otimes} \mu\right)+\sum_{i \geqslant 0} f^{*}\left(\alpha_{i}\right) p_{2}^{i}\left(\left(\xi_{1}+\bar{\xi}_{1}\right) \underset{\mathbb{R}}{\otimes} \mu\right) .
$$

Then by (2.4)

$$
p_{1}\left(\left(\xi_{1}+\bar{\xi}_{1}\right) \underset{\mathbb{R}}{\otimes} \mu\right)=(1 \times p)^{*} p_{1}\left(\left(\xi_{1}+\bar{\xi}_{1}\right) \underset{\mathbb{R}}{\otimes} \Lambda\right)-p_{1}\left(\left(\xi_{1}+\bar{\xi}_{1}\right) \underset{\mathbb{R}}{\otimes} \nu\right),
$$

hence

$$
\begin{aligned}
& \tau^{*}\left(p_{1}\left(\xi_{1} \xi_{2}^{2}+\bar{\xi}_{1} \bar{\xi}_{2}^{2}\right)\right) \\
& =\tau_{2}^{*}\left(p_{1}\left(\left(\xi_{1}+\bar{\xi}_{1}\right) \underset{\mathbb{R}}{\otimes} \mu\right)\right) \\
& \quad+\tau_{2}^{*}\left(\sum_{i \geqslant 0} f^{*}\left(\alpha_{i}\right) p_{2}^{i}\left(\left(\xi_{1}+\bar{\xi}_{1}\right) \underset{\mathbb{R}}{\otimes} \mu\right)\right) \\
& =\tau_{2}^{*}\left((1 \times p)^{*}\left(p_{1}\left(\left(\xi_{1}+\bar{\xi}_{1}\right) \underset{\mathbb{R}}{\otimes} \Lambda\right)\right)\right)-\tau_{2}^{*}\left(p_{1}\left(\left(\xi_{1}+\bar{\xi}_{1}\right) \underset{\mathbb{R}}{\otimes} \nu\right)\right) \\
& \quad+\tau_{2}^{*}\left(\sum_{i \geqslant 0} f^{*}\left(\alpha_{i}\right) p_{2}^{i}\left(\left(\xi_{1}+\bar{\xi}_{1}\right) \underset{\mathbb{R}}{\otimes} \mu\right)\right) \\
& =p_{1}\left(\left(\xi_{1}+\bar{\xi}_{1}\right) \underset{\mathbb{R}}{\otimes} \Lambda\right) \tau_{2}^{*}(1)-\tau_{2}^{*}\left(p_{1}\left(\left(\xi_{1}+\bar{\xi}_{1}\right) \underset{\mathbb{R}}{\otimes} \nu\right)\right) \\
& \quad+\tau_{2}^{*}\left(\sum_{i \geqslant 0} f^{*}\left(\alpha_{i}\right) p_{2}^{i}\left(\left(\xi_{1}+\bar{\xi}_{1}\right) \underset{\mathbb{R}}{\otimes} \mu\right)\right) .
\end{aligned}
$$

Now we have to prove that $\tau_{2}^{*}(1)=1$, the second summand in (3.2) coincides with $x_{1}=p_{1}\left(\xi_{1}+\bar{\xi}_{1}\right)$, and the third summand is zero.

Note that the bundle $\left(\xi_{1}+\bar{\xi}_{1}\right) \otimes_{\mathbb{R}} \nu$ is the pullback of the bundle $\zeta \otimes \eta \rightarrow B \operatorname{Sp}(1) \times$ $B \mathbb{Z} / 2$ along the map $(\pi, f)$. Thus $p_{1}(\zeta \otimes \eta)$ is an element from $M \operatorname{Sp}^{*}(B \mathbb{Z} / 2) \llbracket p_{1}(\zeta) \rrbracket$, hence

$$
p_{1}(\zeta \otimes \eta)=p_{1}(\zeta)+\sum_{i \geqslant 0} \beta_{i} p_{1}^{i}(\zeta)
$$

for some elements $\beta_{i} \in \widetilde{M \mathrm{Sp}}^{*}(B \mathbb{Z} / 2)$. This implies

$$
p_{1}\left(\left(\xi_{1}+\bar{\xi}_{1}\right) \underset{\mathbb{R}}{\otimes} \nu\right)=p_{1}\left(\xi_{1}+\bar{\xi}_{1}\right)+\sum_{i \geqslant 0} f^{*}\left(\beta_{i}\right) p_{1}^{i}\left(\xi_{1}+\bar{\xi}_{1}\right) .
$$

Similarly the bundle $\left(\xi_{1}+\bar{\xi}_{1}\right) \otimes_{\mathbb{R}} \mu$ is the pullback of the bundle $\zeta \otimes \eta(2) \rightarrow$ $B \mathrm{Sp}(1) \times B \mathrm{O}(2)$, where $\eta(2) \rightarrow B \mathrm{O}(2)$ is the universal $\mathrm{O}(2)$-bundle. Hence

$$
p_{2}\left(\left(\xi_{1}+\bar{\xi}_{1}\right) \underset{\mathbb{R}}{\otimes} \mu\right) \in M \operatorname{Sp}^{*}(B \mathrm{~N}) \llbracket p_{1}\left(\xi_{1}+\bar{\xi}_{1}\right) \rrbracket
$$

and for the third summand of (3.2) one has

$$
\sum_{i \geqslant 0} f^{*}\left(\alpha_{i}\right) p_{2}^{i}\left(\left(\xi_{1}+\bar{\xi}_{1}\right) \underset{\mathbb{R}}{\otimes} \mu\right)=\sum_{i \geqslant 0} \gamma_{i} p_{1}^{i}\left(\xi_{1}+\bar{\xi}_{1}\right)
$$

for some $\gamma_{i} \in \widetilde{M S p}^{*}(B N)$.

So using (3.1) one has

$$
\tau^{*}\left(p_{1}\left(\xi_{1} \xi_{2}^{2}+\bar{\xi}_{1} \bar{\xi}_{2}^{2}\right)\right)=p_{1}\left(\left(\xi_{1}+\bar{\xi}_{1}\right) \underset{\mathbb{C}}{\otimes} \zeta^{2}\right) \tau_{2}^{*}(1)-x_{1}\left(\tau_{2}^{*}(1)+1\right)+\sum_{i=0}^{n} \tau_{2}^{*}\left(\delta_{i}\right) x_{1}^{i}
$$

for some $\delta_{i} \in \widetilde{M S p}^{*}(\mathbb{C} P(2 n+1) / I)$. 
It is known from [18, 19] that up to dimension $32, M \mathrm{Sp}_{4 n}$ is torsion free. Motivated by this fact let us assume that $M \mathrm{Sp}_{4 m}$ is torsion free when $m \leqslant 2 n-1$. Then it follows that $M \operatorname{Sp}^{4 k}(\mathbb{H} P(n))$ is torsion free when $k \geqslant 1-n$. Then since the minimal dimension of the elements $\delta_{i}$ from (3.3) is $4-4 n$, it follows from Lemma 2.1] that the third summand of (3.3) restricts to zero in $M \mathrm{Sp}^{*}(\mathbb{H} P(n))$. Also $\tau^{*}(p)(1)=1$ and $\tau^{*}(\pi)(1)=2$.

Thus one obtains from (3.3) and then Lemma 2.4

$$
\phi_{j} p_{1}\left(\left(\xi_{1}+\bar{\xi}_{1}\right) \underset{\mathbb{C}}{\otimes} \zeta_{2}^{2}\right)=\phi_{j} \tau^{*}\left(p_{1}\left(\xi_{1} \xi_{2}^{2}+\bar{\xi}_{1} \bar{\xi}_{2}^{2}\right)\right)=0
$$

This proves Theorem 1.19).

For the proof of b) note that it follows from Lemma 2.1 that for the bundle $\pi \times 1$ $\left.=\pi \times 1_{\mathbb{H} P(n)}\right)$ one has

$$
\begin{aligned}
&(\pi \times 1)^{*} \tau_{\pi \times 1}^{*}\left(p_{1}\left(r \xi_{1} \underset{\mathbb{R}}{\otimes} \zeta_{2}\right)\right)=(1+I)^{*}\left(p_{1}\left(\left(\xi_{1}+\bar{\xi}_{1}\right) \underset{\mathbb{C}}{\otimes} \zeta_{2}\right)\right) \\
&=2 p_{1}\left(\left(\xi_{1}+\bar{\xi}_{1}\right) \underset{\mathbb{C}}{\otimes} \zeta_{2}\right)=(\pi \times 1)^{*} p_{1}\left(\left(\zeta_{1} \underset{\mathbb{R}}{\otimes} \zeta_{2}\right)\right) .
\end{aligned}
$$

Then by (2.5) any element from $\operatorname{ker}(1 \times \pi)^{*}$ is divisible by $e(\Lambda)$. On the other hand by hypothesis $M \mathrm{Sp}^{4 k}(\mathbb{H} P(n))$ is torsion free for $k \geqslant 1-n$. Hence one concludes that restriction of the homomorphism $(\pi \times 1)^{*}$ to $M \mathrm{Sp}^{*}\left(\mathbb{H} P(n)^{2}\right)$ is a monomorphism, thus in $M \operatorname{Sp}^{4}\left(\mathbb{H} P(n)^{2}\right)$ one has

$$
p_{1}\left(\zeta_{1} \underset{\mathbb{R}}{\otimes} \zeta_{2}\right)=\tau_{\pi \times 1}^{*}\left(p_{1}\left(r \xi_{1} \underset{\mathbb{R}}{\otimes} \zeta_{2}\right)\right) .
$$

Now since Proposition 2.5 says that the right-hand side is zero after multiplication by $\phi_{j}$, this completes the proof of Theorem 1.1

\section{Proof of Corollary 1.2}

Let $h: \pi_{*}(M \mathrm{Sp}) \rightarrow H_{*}(M \mathrm{Sp})=\mathbb{Z}\left[q_{1}, q_{2}, \ldots\right]$ be the Hurevicz homomorphism. Since $\pi_{4 n}(M \mathrm{Sp})$ is torsion free for small $n$ (see [16, 18, 19]), the Hurevicz homomorphism is a monomorphism in these dimensions. So in low dimensions $4 n$ the Hurevicz homomorhism determines all relations. Our aim here is to express the coefficients $a_{k l m}$ from the Introduction through the generators $x$-es.

Values of the Hurevicz homomorphism on these $a_{k l m}$ are calculated in [10]. In low dimensions one has

$$
\begin{aligned}
& h\left(a_{100}\right)=h\left(a_{010}\right)=h\left(a_{001}\right)=4, \\
& h\left(a_{200}\right)=h\left(a_{020}\right)=h\left(a_{002}\right)=0, \\
& h\left(a_{110}\right)=h\left(a_{101}\right)=h\left(a_{011}\right)=24 q_{1}, \\
& h\left(a_{111}\right)=360 q_{2}, \\
& h\left(a_{210}\right)=\ldots=h\left(a_{012}\right)=60 q_{2}-24 q_{1}^{2}, \\
& h\left(a_{300}\right)=\ldots=h\left(a_{003}\right)=0, \\
& h\left(a_{220}\right)=\ldots=h\left(a_{022}\right)=280 q_{3}-120 q_{1} q_{2}+24 q_{1}^{3}, \\
& h\left(a_{310}\right)=\ldots=h\left(a_{013}\right)=112 q_{3}-96 q_{1} q_{2}+48 q_{1}^{3}, \\
& h\left(a_{211}\right)=\ldots=h\left(a_{112}\right)=1680 q_{3}-360 q_{1} q_{2}, \\
& h\left(a_{122}\right)=\ldots=h\left(a_{122}\right)=75600 q_{4}-3360 q_{1} q_{3}+360 q_{1}^{2} q_{2}, \\
& h\left(a_{410}\right)=\ldots=h\left(a_{140}\right)=180 q_{4}-360 q_{1} q_{3}+420 q_{1}^{2} q_{2}-120 q_{2}^{2}-120 q_{1}^{4} .
\end{aligned}
$$


Further, the Hurevicz images of generators of $M S p$ are calculated in [16]. Namely, $h\left(M \mathrm{Sp}_{4 k}\right) \subset H_{4 k}(M \mathrm{Sp})$ has the following generators:

$$
\begin{aligned}
k=1: & 24 q_{1}, \\
k=2: & 20 q_{2}-8 q_{1}^{2}, 144 q_{1}^{2}, \\
k=3: & 56 q_{3}-72 q_{1} q_{2}+24 q_{1}^{3}, 120 q_{1} q_{2}-48 q_{1}^{3}, 3456 q_{1}^{3}, \\
k=4: & 12 q_{4}-24 q_{1} q_{3}-8 q_{2}^{2}+28 q_{1}^{2} q_{2}-8 q_{1}^{4}, 50 q_{2}^{2}+168 q_{1} q_{3}-256 q_{1}^{2} q_{2}+80 q_{1}^{4}, \\
& 100 q_{2}^{2}-80 q_{1}^{2} q_{2}+16 q_{1}^{4}, 2880 q_{1}^{2} q_{2}-1152 q_{1}^{4}, 20736 q_{1}^{4} .
\end{aligned}
$$

Thus one concludes that the elements $a_{011}, a_{111}, a_{022}, a_{122}, a_{112}, a_{120}, a_{140}$ are generators as in the Introduction.

Remark 1. In terms of $2 x_{i}$, the generators of $M \mathrm{Sp}_{4 n}$ from [16], one has modulo $2 M \mathrm{Sp}_{*}: 2 x_{1}=a_{011}, 2 x_{2}=a_{012}, 2 x_{3}=a_{022}, x_{4}=a_{014}, x_{1}^{2}=a_{111}$, etc.

Remark 2. Alternatively, images of the elements $a_{i j k}$ in complex cobordism $M \mathrm{U}_{*}$ can be calculated in terms of two-valued formal groups:

$$
\mu^{*}\left(p_{1}\left(\zeta_{1} \underset{\mathbb{C}}{\otimes} \zeta_{2} \underset{\mathbb{C}}{\otimes} \zeta_{3}\right)\right)=\Theta_{1}\left(x_{1}, Y^{+}\right)+\Theta_{1}\left(x_{1}, Y^{-}\right)
$$

where $Y^{+}+Y^{-}=\Theta_{1}\left(x_{2}, x_{3}\right), Y^{+} Y^{-}=\Theta_{2}\left(x_{2}, x_{3}\right) ; \Theta_{1}$ and $\Theta_{2}$ are the coefficients of the two-valued formal group [5] and $\mu^{*}$ is the obvious map from the symplectic cobordism theory to the complex cobordism theory.

Let us now consider Corollary[1.2. From Theorem[1.1 $)$, in $M \mathrm{Sp}^{*}(\mathbb{H} P(4) \times \mathbb{H} P(4)$ one has a relation of the form

$$
\begin{aligned}
\phi_{j}\left(a_{011} x_{2}^{2}+a_{111} x_{1} x_{2}^{2}+a_{022} x_{2}^{4}+a_{211} x_{1}^{2} x_{2}^{2}+a_{122} x_{1} x_{2}^{4}+\ldots\right) & \\
& =\left(\phi_{0} x_{1}+\sum_{1 \leqslant i \leqslant n} \phi_{i} x_{1}^{2 i}\right) b\left(x_{1}, x_{2}\right)
\end{aligned}
$$

for some element $b\left(x_{1}, x_{2}\right) \in M \mathrm{Sp}^{*}\left(\mathbb{H} P(n)^{2}\right)$. Then by the equality of the coefficients at the monomials $x_{1} x_{2}^{2}, x_{1}^{2} x_{2}^{2}$ and $x_{1} x_{2}^{4}$ one obtains assertions $\mathrm{b}$ ) and c) of Corollary [1.2.

Similarly from Proposition 2.5 one has

$$
\phi_{j}\left(a_{110} x_{2}^{2}+a_{120} x_{1} x_{2}^{2}+a_{220} x_{1}^{2} x_{2}^{2}+a_{140} x_{1} x_{2}^{4}+\ldots\right)=0,
$$

and hence assertion a) of Corollary 1.2 is valid.

Proposition 4.1. In dimension 32 there is an element $y_{4}^{2}$ such that $\phi_{2} y_{4}^{2}$ does not belong to the ideal generated by $\phi_{0}$ and $\phi_{1}$. Moreover $\phi_{2^{i}} y_{4}^{2}$ does not belong to the ideal generated by $\phi_{0}, \phi_{1}, \ldots, \phi_{2^{i-1}}, i \geqslant 1$.

Proof. It follows from the calculations of the symplectic cobordism ring made in [18, 19].

\section{REFERENCES}

[1] M. F. Atiyah, Characters and cohomology of finite groups, Publ. Math. of the I.H.E.S., 9 (1961) 23-64 MR 26:6228

[2] J. C. Becker, P. H. Gottlieb, The transfer map and fibre bundles, Topology, 14 (1975) 1-15

[3] J. M. Boardman, Stable homotopy theory (mimeographed), University of Warwick (1966)

[4] K. Brown, Cohomology of groups. Graduate Texts in Mathematics, 87. Springer-Verlag, New York-Berlin, 1982. 306 pp. MR 83k:20002 
[5] V. M. Buchstaber, Characteristic classes in cobordisms and topological applications of theories of one and two valued formal groups, Itogi nauki i tekniki, 10 (1977) 5-178

[6] A. Dold, The fixed point transfer of fibre preserving maps, Math. Z., 148 (1976) 215-244 MR 55:6416

[7] M. Feshbach, The transfer and compact Lie groups, Trans. Amer. Math. Soc. (1979, July), 251, 139-169 MR 80k:55049

[8] V. G. Gorbunov, Symplectic cobordism of projective spaces, Math. Sbornik, 181 (1990) 506-520 MR 91i:55006

[9] V. Gorbunov, N. Ray, Orientations of Spin bundles and symplectic cobordism. Publ. Res. Inst. Math. Sci., 28 (1992), no. 1, 39-55 MR 93e:55008

[10] M. Imaoka, Symplectic Pontrjagin numbers and homotopy groups of $M \operatorname{Sp}(n)$. Hiroshima Math. J., 12 (1982) no. 1, 151-181 MR 83e:57026

[11] D. S. Kahn, S. B. Priddy, Applications of the transfer to stable homotopy theory, Bull. Amer. Math. Soc., 78 (1972) 981-987 MR 46:8220

[12] J. Milnor, On the cobordism ring $\Omega^{*}$ and a complex analogue. I. Amer. J. Math., 82 (1960) 505-521 MR 22:9975

[13] R. Nadiradze, Characteristic classes in $\mathrm{SC}^{*}$ theory and their applications, I. Baku Intern. Top. Conf. Abstracts (1987), 213; II. Preprint, Tbilisi Razmadze Math. Inst. (1991), 1-11; III. Preprint, Heidelberg, 58 (1993) 1-21

[14] S. P. Novikov, Homotopy properties of Thom complexes. Mat. Sb. (N.S.), 57 (99) (1962) 407-442. (Russian) MR 28:615

[15] N. Ray, Indecomposables in TorsMSp ${ }_{*}$, Topology, 10 (1971) 261-270 MR 45:9342

[16] N. Ray, The symplectic bordism ring, Proc. Camb. Phil. Soc., 71 (1972) 271-282 MR 44:7567b

[17] F. W. Roush, On some torsion classes in symplectic bordism, Preprint.

[18] V. V. Vershinin, Computation of the symplectic cobordism ring in dimensions less than 32 and the non-triviality of the majority of the triple products of Ray's elements, Sibirsk. Math. Zh., 24 (1983) 50-63

[19] V. V. Vershinin, Cobordisms and spectral sequences. Translations of Mathematical Monographs, 130. AMS, Providence, RI, 1993. MR 94j:55006

[20] V. V. Vershinin, A. L. Anisimov, A series of elements of order 4 in the symplectic cobordism ring. Canad. Math. Bull., 38 (1995), no. 3, 373-381 MR 96i:55011

Razmadze Mathematical Institute, Tbilisi 380093, Republic of Georgia

E-mail address: maxo@rmi.acnet.ge

Razmadze Mathematical Institute, Tbilisi 380093, Republic of Georgia

E-mail address: jib@rmi.acnet.ge

DÉPARTEment des sciences mathématiques, CNRS, UMR 5030 (GTA), Université Montpellier II, place Eugéne Bataillon, 34095 Montpellier Cedex 5, France - And - Institute of Mathematics, Novosibirsk 630090, Russian Federation

E-mail address: vershini@darboux.math.univ-montp2.fr

E-mail address: versh@math.nsc.ru 